\title{
Gender Differences and the Moderating Role of Stress Mindset in Stressful Life Events and Well-being
}

\author{
Luoyao Zhang
}

\author{
The Gilbert School 200 Williams Avenue CT, US \\ kristoffferzhang@gmail.com
}

\begin{abstract}
Stress is defined in psychology as a feeling of emotional tension and pressure. Stress is not only a cognitive and behavioral experiential process but also closely associated with many psychiatric disorders. Gender is an important biological determinant of a person's vulnerability to psychosocial stress; therefore, stress, stress reactions, and stress-related disorders all exhibit gender checks. At the same time, when faced with stress and stress reactions, different people tend to have different stress mindsets: some people have a stress-enhancing mindset, which believes that stress has positive cognitive, psychological, emotional, and hormonal effects, while others have a stress-weakening mindset, which believes that stress has negative effects on all aspects. In order to better investigate individual differences in stress and stressful attitudes, and to further explore the relationship between stressful attitudes and individuals' past experiences, health status, and life satisfaction, a total of 400 (200 male and 200 female) students aged 19 to 24 years from a university in the United States will be recruited to complete a series of self-reported questionnaires on stressful life events, stressful attitudes, general health, and life satisfaction. Based on the gender differences in stress, stress response, and the application of gender differences in disease diagnosis and treatment, this study aims to explore the gender differences in stress states of mind, so as to deepen our understanding of individual differences in stress and stress states of mind, and to lay a theoretical foundation for clinical practitioners to make more accurate diagnoses and propose more targeted treatment strategies for stress-related diseases.
\end{abstract}

Keywords: Stress mindset, Stressful life events, Well-being, Gender differences

\section{INTRODUCTION}

As a psychological term, the word stress was born in the last century and was first introduced by psychologist Hans Selye. Stress is a cognitive and behavioral experience, which leads to different reactions to stress and different levels of stress in different people [1]. At the same time, stress has been associated with many psychiatric disorders, including schizophrenia, anxiety, depression, and addictive behaviors [2]. Research suggests that stress arises from stressor, which is generated by both early life events and acute adult events. Stressors lead to stress and stress could bring about both neurobiological and psychological changes to our bodies [3]. While some events are considered stressful for all people, individual differences, differences in resilience, and differences in vulnerability are recognized as the most studied topics in the field of stress research [4].
Gender is an important determinant of human health, almost all types of physical and psychological disorders have their unique gender profiles. In general, men are more susceptible to infectious diseases, hypertension, aggressive behavior, and substance abuse while disorders such as autoimmune diseases, chronic pain, depression, and anxiety disorders are relatively more prevalent in women [5], [6]. In addition to disease, males and females exhibit significantly different stress responses psychologically and physiologically [7]. Currently, the assessment of sex differences in stress reactivity relies primarily on measuring physiological responses to acute stressors in a laboratory and the sympathetic nervous system (e.g., heart rate and blood pressure) [5]. Researchers have found that women's tendency to develop depression is associated with an impaired feedback effect of cortisol on HPA arousal. Compared with adult women, studies have found greater acute HPA and autonomic responses in adult men, aided by standard performance-related psychosocial stressors (e.g., public speaking), and this difference is likely due 
to female sex hormones that impair sympathetic and HPA responsiveness, thereby delaying cortisol feedback to the brain and thereby reducing or curtailing stress response [6], [8]. Performance stress paradigm experiments and neuroimaging studies also confirm that acute HPA and autonomic responses are greater in men than in women [8]. Neuroimaging studies have found that women exhibit a greater degree of emotional "flashbacks" (melancholic thinking) after completing stressful tasks compared to men; this phenomenon may be caused by sustained cingulate [9]. However, similar neuroimaging studies did not produce consistent results. From a stressor perspective, experiments comparing different stressors found that sex differences in stress responses or differences in cortisol elevation were only seen in achievement task stressor experiments; when experiments were conducted using a social exclusion task, researchers did not observe significant sex differences or differences in cortisol elevation [10]. Some researchers have found that women are more likely than men to be negatively affected by interpersonal events by comparing the nature of the stressors [11]; in such stressful practices, men and women exhibit different stress responses: men are more inclined to "fight or flight" while women are more inclined to "get close to friends."[12] Given that physiological stress responses are often closely linked to activation of the sympathetic nervous system and the HPA axis, researchers have recently conducted neuroendocrine and behavioral studies. It was found that as women are more inclined to attachment and caregiving, the response to stress on this premise buffers sympathetic stimulation while also curbing HPA axis activation [11].

Mindset is defined in psychology as a mental framework or lens. Mindset is the way we can selectively organize and encode information that directs different individuals to their own unique understandings, which in turn guides people to act and react accordingly [13]. Although reducing and avoiding stress is nearly impossible for individuals, the processes by which different individuals cope with stress are complex and variable. In recent years, psychologists have further explored the effects of mindset on intelligence, mood, and health [14]-[17]. Based on these studies it is easy to see that mindset is indeed a better strategy when it comes to stress management. In general, stress mindset is conceptualized as the extent to which an individual holds the mindset that stress is either enhancing or debilitating. On the one hand, individuals who believe that stress has enhancing consequences (referred as "stress-is-enhancing") see that stress may positively impact on their performance and productivity, health, and well-being, and learning and growth. On the other hand, those who holds the mindset that stress has debilitating consequences (referred as "stress-is-debilitating") belive that stress will produce only negative impacts on various outcomes [18]. Crum et al. have shown that changes in mindset can affect health through indirect behavioral changes as well as direct physiological changes; therefore, one's stressful state of mind can significantly affect one's health-related conditions [19], [20]. Stressful mindsets can shape both stress responses and affect intelligence [13] and aging [21]. Researchers have now identified two stress mindsets; if a person holds a stress-as-debilitating mindset, then his or her primary motivation is to avoid or manage stress to prevent debilitating. On the other hand, if a person holds a stress-as-enhancement mindset, then the primary motivation is to accept and use stress to achieve these enhanced outcomes [18]. Research has also shown that threat and challenging evaluations are also moderated by stress mindsets: individuals who hold a stress-as-enhancement mindset are more likely to improve their responses to threat evaluations, whereas individuals who hold a stress-as-debilitation mindset are more likely to worsen their translation of threat evaluations. In Crum et al.'s experiment, participants who perceived stress-as-enhancement spent more time focusing on happy faces than those who perceived stress-as-weakening. In the DHEAS experiment, participants who had a stress-as-enhancement mindset showed a more positive physiological response. These studies demonstrate that the positive cognitive and affective effects of threatening and challenging evaluations generally only occur when handled with a stress-as-enhanced mindset; also, stress and an enhanced mindset can help improve subjects' physiological responses in response to challenging and threatening stress by enhancing the DHEAS. On the other hand, a person's stress mindset is not always static. Experiments have demonstrated that interventions such as images or words can be effective in orienting subjects to different information that can change their stress mindset and ultimately have a corresponding impact in subjects self-reported health and work performance [18], [19], [22]. Therefore, changing one's attitude toward stress (stress mindset) is one of the ways in which one can effectively influence and change one's stress response [23]- [25]. Given that the stress response can be associated with the manifestation of both various types of physical and psychological disorders, we need to explore closer the individual differences, individual resilience and vulnerability in the alteration of the stress mindset and the altered stress response it entails.

In conclusion, stress significantly affects each of us in our daily lives; it is not only a simple personal problem, but also a profound social problem. Experiments have shown that stress mindset can significantly improve the emergency response to stress. Exploring the gender differences in the modifiability of stress mindset and stress patterns and their influencing factors is not only important for deepening the understanding of stress and stress mindset, but also 
beneficial for clinical workers to make more accurate diagnosis and propose more targeted treatment strategies for stress-related diseases. Based on the significant gender differences in the HPA axis, stress response, and psychiatric disorders, we hypothesize that stress mindset and the moderation effect of stress mindset on the relationship between stress and well-being differ among male and female.

\section{MATERIALS AND METHODS}

\subsection{Subjects}

We plan to recruit 200 men and 200 women ranging in age from 19 to 24 years old from a university to do the surveys. The entire questionnaire will last approximately 45 minutes. Written and verbal instructions will be provided to the subjects. At the end of the survey, each participant will be paid $\$ 15$.

\subsection{Measures}

\section{Stressful Life Events (LEC) [28]}

Stressful life events will be measured using the Life Events Checklist. The Life Events Checklist (LEC) was developed at the National Center for PTSD to be administered concurrently with the clinician-administered Posttraumatic Stress Disorder Scale (CAPS) to assess exposure to PTEs. The LEC is used to assess the respondent's experience of various traumatic experiences, and the CAPS is then used to identify the index event (worst or most salient), clarify the specific nature of the recognized event, determine whether the event meets the definition of trauma criteria-A as described in the DSM-IV (APA, 1994), and to assess the presence and severity of posttraumatic symptoms resulting from the index experience. The scale consists of 17 potential treatment events (PTEs). For each PTEs, respondents rated their experience of the event on a nominal scale of 5 ( 1 =happened to me, 2=witnessed it, 3=understood it, 4=unsure, 5=not applicable). We summed the selected items to create an index of the level of the stressful life event, with higher scores indicating higher levels of the stressful life event.

\section{Stress Mindset [18]}

This eight-item measure was developed by Crum, Salovey, and Achor (2013) to address the extent to which a person adopts a mindset that is enhanced or diminished by the effects of stress. The items listed below assessed participants' general stress mindset (e.g., "The effects of stress are negative and should be avoided"), as well as signs and symptoms related to the enhancing and debilitating consequences of stress in terms of health and vitality, learning and growth, and performance and productivity (e.g., "Experiencing stress improves health and vitality"). Participants rated items on a five-point scale ranging from $0=$ strongly disagree to $4=$ strongly agree. SMM scores were obtained by reverse scoring the four negative items (denoted by *) and then averaging all eight items. higher SMM scores represent a stress-enhancing mindset.

\section{General Health Survey [27]}

The EQ-5D EuroQol Five Dimensions Questionnaire, a standardized set of scales to measure health status, was developed by the European Society for Quality of Life (EuroQol) to provide a simple, universal measure of health. The EQ-5D series is designed to describe and evaluate the health status of patients in various disease areas. The scales have been used in clinical trials, population-based studies and have been shown to have good reliability, validity and sensitivity in a variety of disease areas and populations.

The EQ-5D consists of two main components: the EQ-5D Descriptive System and the EQ-5D Visual Analogue Scale (VAS), which describes health status in five dimensions: Mobility, Self-care, and Activities of Daily Living. Self-care), daily activities (Usual Activities), pain/discomfort (Pain/Discomfort), and anxiety/depression (Anxiety/Depression). The questionnaire asked respondents to select the most appropriate option in each dimension according to their health status. Options were coded by numbers 1, 2, 3, 4, and 5.1 indicated no difficulty at all, 2 indicated a little difficulty, 3 indicated moderate difficulty, 4 indicated severe difficulty, and 5 indicated unable to perform/have very severe difficulty. In the assessment section, respondents assessed their general health status using a visual analog scale (EQ-VAS). The EQ-VAS is a vertical scale on which the respondent's self-rated health status is recorded. The scale is $0-100$, with 0 indicating "the worst health you can imagine" and 100 indicating "the best health you can imagine. Respondents' self-rated information can be used as a quantitative indicator of health outcomes. The health description system of the EQ-5D scale generates a five-digit health status, which can be visually reflected in five health dimensions.

\section{Satisfaction with Life Scale [26]}

The Satisfaction with Life Scale (SWLS) consists of five items that measure subjects' satisfaction with their lives (e.g., "In most respects, my life is close to my ideal"). Participants reported their level of agreement with each statement. On a scale of 1 (completely disagree) to 7 (completely agree), participants reported their level of agreement with each statement. The mean of all items was calculated, with higher scores indicating higher life satisfaction. Scores correlated with other measures of subjective well-being and were correlated with specific personality traits. 


\subsection{Data Analyses}

We will calculate the means, standard deviations, and correlation coefficients for the main variables based on the scale results.

We will investigate the moderating effects of stress mindset and gender on the relationship between stressful life events and the two well-being indicators ("general health" "life satisfaction") using a series of hierarchical multiple regressions. In each model, demographic variables will be controlled. Gender ( $\operatorname{girl}=0$, boy $=1$ ), stressful life events, and the stress mindset will be entered as the main predictors. We will examine the two-way terms (interactions between "stressful life events and general health", "stress mindset and life satisfaction", "stress mindset and gender"). We will also analyze the three-way interaction terms for "stress mindset, stressful life events, and general health", "stress mindset, stressful life events, and life satisfaction", "stress mindset, life satisfaction, and gender", "stress mindset, general health, and gender"

\section{EXPECTED RESULTS}

\subsection{Stress mindset and general health}

If SMM is positively correlated with EQ5D, it means that the better the mindset, the higher the depressive tendency; if SMM is negatively correlated with EQ5D, it means that the better the mindset, the lower the depressive tendency; if SMM is not correlated with EQ5D, it means that there is no significant relationship between mindset and depressive tendency

\subsection{Stress mindset and life satisfaction}

If SMM is positively correlated with SWLS, it means that the better the mindset, the higher the life satisfaction; if SMM is negatively correlated with SWLS, it means that the better the mindset, the lower the life satisfaction; if SMM is not correlated with SWLS, it means that mindset has no significant relationship with life satisfaction

\subsection{Stress mindset, stressful life events, and general health}

The tendency to depression predicted by stressful life events, stressful mindset by stratified multiple regression.

If $\mathrm{p}<0.05$, it means that stress i.e. enhanced mindset protects against depressive tendencies by mitigating the negative effects of stressful life events

If $\mathrm{p}>0.05$, then it means that stressful mindset, stressful events have no significant effect on depressive tendency

\subsection{Stress mindset, stressful life events, and life satisfaction}

Life satisfaction predicted by stressful life events, stressful mindset by stratified multiple regression.

If $\mathrm{p}<0.05$, it means that stress i.e. enhanced mindset improves life satisfaction by mitigating the negative effects of stressful life events

If $p>0.05$, it means that stressful mindset and stressful events have no significant effect on life satisfaction

\subsection{Stress mindset and gender}

If there is a significant difference between the mean of SSM scale for males and females and males are higher than females, it means that males generally hold stress-is-enhancing mindset

If there is a significant difference between males and females on the SSM scale and males are lower than females, then females generally hold a stress-is-enhancing mindset

If there is no significant difference between the means of SSM scale males and females, then it means that males and females do not differ significantly in their mindset.

\subsection{Stress mindset, life satisfaction, and gender}

If $p<0.05$ in simple slope analysis between mindset and life satisfaction for female-> female mindset is positively correlated with life satisfaction, i.e., among females, the better the mindset the higher the life satisfaction

If $p<0.05$ in simple slope analysis between mindset and life satisfaction for male $\rightarrow$ Male mindset is positively correlated with life satisfaction, i.e., among males, the better the mindset, the higher the life satisfaction

If $\mathrm{p}>0.05$ in simple slope analysis between mindset and life satisfaction for male-> that is, among males, mindset is not related to life satisfaction

If $p>0.05$ in simple slope analysis between mindset and life satisfaction for female-> that is, among females, mindset is not related to life satisfaction

\subsection{Stress mindset, general health, and gender}

If $\mathrm{p}<0.05$ in simple slope analysis between mindset and depression for female-> the negative association between mindset and depression tendency for females, i.e., the better the mindset the lower the depression tendency in females 
If $p<0.05$ in simple slope analysis between mindset and life satisfaction for male-> Male mindset is negatively correlated with depression tendency, i.e., among males, the better the mindset, the lower the depression tendency

If $\mathrm{p}>0.05$ in simple slope analysis between mindset and life satisfaction for male- $>$ that is, among males, mindset is not related to depressive tendency

If $p>0.05$ in simple slope analysis between mindset and life satisfaction for female-> that is, among females, mindset is not associated with depressive tendency

\section{CONCLUSION}

In sum, this research proposal intent to study gender differences and the moderating role of stress mindset in stressful life events and well-being. Through our experimental design, we intend to first validate stress mindset whether stress mindset as a predictor for general health and life satisfaction by investigating the relationship between SSM, EQ5D, and SWLS. Secondly, we want to explore whether there is a gender difference in stress mindset by analyzing the relationship between SSM result and responder's gender. As mentioned in the introduction, since almost all types of physical and psychological disorders have their unique gender profiles, we intend to investigate whether the moderation effect of stress mindset towards different scales of stressful life event could also have its unique gender profile. And if so, does this profile apply to the moderation effect of stress mindset towards general health and life satisfaction. To date, there are very few studies examining gender differences in the effects of the stress mindset, and there is no study that examined the gender differences in the moderation effects of stress mindset toward stressful life event, general health, and life satisfaction. Therefore, this study will extend the field of stress and stress mindset research.

\section{REFERENCES}

[1] Selye, H. (1973). The Evolution of the Stress Concept: The originator of the concept traces its development from the discovery in 1936 of the alarm reaction to modern therapeutic applications of syntoxic and catatoxic hormones. American Scientist,61(6), 692-699. Retrieved July 20, 2021, from www.jstor.org/stable/27844072. Accessed 20 July 2021.

[2] Sinha, R. (2008). Chronic Stress, Drug Use, and Vulnerability to Addiction. Annals of the New York Academy of Sciences,1141(1), 105-130. doi:10.1196/annals.1441.030

[3] Schneiderman, N., Ironson, G., \& Siegel, S. D. (2005). Stress and Health: Psychological, Behavioral, and Biological Determinants. Annual
Review of Clinical Psychology,1(1), 607-628. doi:10.1146/annurev.clinpsy.1.102803.144141

[4] Godoy, L. D., Rossignoli, M. T., Delfino-Pereira, P., Garcia-Cairasco, N., \& Umeoka, E. H. (2018). A Comprehensive Overview on Stress Neurobiology: Basic Concepts and Clinical Implications. Frontiers in Behavioral Neuroscience, 12. doi:10.3389/fnbeh.2018.00127

[5] Holden, C. (2005). Sex and the Suffering Brain. Science,308(5728), 1574-1574. doi:10.1126/science.308.5728.1574

[6] Kudielka, B. M., \& Kirschbaum, C. (2005). Sex differences in HPA axis responses to stress: A review. Biological Psychology,69(1), 113-132. doi:10.1016/j.biopsycho.2004.11.009

[7] Lundberg, U. (2005). Stress hormones in health and illness: The roles of work and gender. Psychoneuroendocrinology,30(10), 1017-1021.

[8] Kajantie, E., \& Phillips, D. (2006). The effects of sex and hormonal status on the physiological response to acute psychosocial stress. Psychoneuroendocrinology,31(2), 151-178. doi:10.1016/j.psyneuen.2005.07.002

[9] Papadakis, A. A., Prince, R. P., Jones, N. P., \& Strauman, T. J. (2006). Self-regulation, rumination, and vulnerability to depression in adolescent girls. Development and Psychopathology,18(03). doi:10.1017/s0954579406060408

[10] Dickerson, S. S., \& Kemeny, M. E. (2004). Acute Stressors and Cortisol Responses: A Theoretical Integration and Synthesis of Laboratory Research. Psychological Bulletin,130(3), 355-391. doi:10.1037/0033-2909.130.3.355

[11] Cyranowski, J. M., Frank, E., Young, E., \& Shear, M. K. (2000). Adolescent Onset of the Gender Difference in Lifetime Rates of Major Depression. Archives of General Psychiatry,57(1), 21. doi:10.1001/archpsyc.57.1.21

[12] Biobehavioral Responses to Stress in Females: Tend-and-Befriend, Not Fight-or-Flight. (2002). Foundations in Social Neuroscience. doi:10.7551/mitpress/3077.003.0048

[13] Dweck, C. S. (2008). Can Personality Be Changed? The Role of Beliefs in Personality and Change. Current Directions in Psychological Science,17(6), 391-394. doi:10.1111/j.1467-8721.2008.00612.x

[14] Taylor, S. E., \& Gollwitzer, P. M. (1995). Effects of mindset on positive illusions. Journal of Personality 
and Social Psychology,69(2), 213-226. doi:10.1037/0022-3514.69.2.213

[15] Gollwitzer, P. M. (1999). Implementation intentions: Strong effects of simple plans. American Psychologist,54(7), 493-503. doi:10.1037/0003-066x.54.7.493

[16] Crum, A. J., \& Langer, E. J. (2007). Mind-Set Matters. Psychological Science,18(2), 165-171. doi:10.1111/j.1467-9280.2007.01867.x

[17] Liberman, V., Samuels, S. M., \& Ross, L. (2004). The Name of the Game: Predictive Power of Reputations versus Situational Labels in Determining Prisoner's Dilemma Game Moves. Personality and Social Psychology Bulletin,30(9), 1175-1185. doi:10.1177/0146167204264004

[18] Crum, A. J., Salovey, P., \& Achor, S. (2013). Rethinking stress: The role of mindsets in determining the stress response. Journal of Personality and Social Psychology,104(4), 716-733. doi:10.1037/a0031201

[19] Crum, A. J., Corbin, W. R., Brownell, K. D., \& Salovey, P. (2011). "Mind over milkshakes: Mindsets, not just nutrients, determine ghrelin response": Correction to Crum et al. (2011). Health Psychology,30(4), 429-429. doi:10.1037/a0024760

[20] Lovallo, W. R. (1997). Stress and Health: Biological and Psychological Interactions. doi:10.4135/9781071801390

[21] Levy, B. R., \& Myers, L. M. (2004). Preventive health behaviors influenced by self-perceptions of aging. Preventive Medicine,39(3), 625-629. doi:10.1016/j.ypmed.2004.02.029

[22] Blackwell, L. S., Trzesniewski, K. H., \& Dweck, C. S. (2007). Implicit Theories of Intelligence Predict Achievement Across an Adolescent Transition: A Longitudinal Study and an Intervention. Child Development,78(1), 246-263. doi:10.1111/j.1467-8624.2007.00995.x

[23] Paunesku, D., Walton, G. M., Romero, C., Smith, E. N., Yeager, D. S., \& Dweck, C. S. (2015). Mind-Set Interventions Are a Scalable Treatment for Academic Underachievement. Psychological Science,26(6), 784-793. doi:10.1177/0956797615571017

[24] Tamir, M., John, O. P., Srivastava, S., \& Gross, J. J. (2007). Implicit theories of emotion: Affective and social outcomes across a major life transition. Journal of Personality and Social Psychology,92(4), 731-744. doi:10.1037/0022-3514.92.4.731
[25] Walton, G. M. (2014). The New Science of Wise Psychological Interventions. Current Directions in Psychological $\quad$ Science,23(1), doi:10.1177/0963721413512856

[26] Diener, E., Emmons, R. A., Larsen, R. J., \& Griffin, S. (1985). The Satisfaction With Life Scale. Journal of Personality Assessment,49(1), 71-75. doi:10.1207/s15327752jpa4901_13

[27] Balestroni, G., \& Bertolotti, G. (2015). EuroQol-5D (EQ-5D): An instrument for measuring quality of life. Monaldi Archives for Chest Disease,78(3). doi:10.4081/monaldi.2012.121

[28] Gray, M. J., Litz, B. T., Hsu, J. L., \& Lombardo, T. W. (2004). Psychometric Properties of the Life Events Checklist. Assessment,11(4), 330-341. doi:10.1177/1073191104269954 